\title{
Left main coronary artery and right pulmonary vein compression by a large pulmonary artery aneurysm
}

\author{
V Decuypere, M Delcroix, W Budts
}

Heart 2004;90:e21 (http://www.heartjnl.com/cgi/content/full/90/4/e21). doi: 10.1136/hrt.2003.029694

Pulmonary artery aneurysms are uncommon and may be associated with significant morbidity and mortality. The unique combination of left main coronary artery and upper right pulmonary vein compression by a large pulmonary artery aneurysm is reported. Furthermore, the aetiology, clinical manifestations, complications, diagnostic approach, and possible therapeutic interventions are briefly discussed.

$\mathrm{P}$ ulmonary artery aneurysms (PAAs) are uncommon. Depending on their size and location, they may lead to complications such as extrinsic compression of the left main coronary artery and the upper right pulmonary vein, as we describe in our case. Other complications are thrombus formation, secondary thromboembolic phenomena, and rupture. Therefore, awareness of this condition and its diagnosis are very important to prevent these problems.

\section{CASE REPORT}

A 38 year old woman, born in Afghanistan, had suffered from effort related dyspnoea since childhood. Ten years previously, she had undergone explorative thoracic surgery because of a thoracic mass on chest radiography. Perioperatively, dilated pulmonary arteries were found and a conservative treatment was followed. She was treated with warfarin because of a documented thrombus in the right pulmonary artery.

At presentation she had progressive dyspnoea (New York Heart Association class III-IV). Physical examination showed a cachectic woman of $41 \mathrm{~kg}$. There were no signs of cyanosis or clubbing. Blood pressure was $110 / 80 \mathrm{~mm} \mathrm{Hg}$, pulse rate 110 beats/min, and respiratory rate 24 breaths/min. Clinical cardiac examination showed a bulging precordium with a palpable thrill located at the left border of the sternum. A grade $4 / 6$ systolic murmur along the left sternal border and a grade 4/6 diastolic murmur in the pulmonary area were heard. End expiratory wheezing was present. Jugular venous pressure was increased. There was no hepatomegaly or peripheral oedema.

A resting ECG showed sinus tachycardia of 117 beats/min, $\mathrm{P}$ pulmonale, and right ventricular hypertrophy. On chest radiography bilateral hilar masses were found (fig 1A). Computed tomography showed important aneurysmal dilatation of both pulmonary arteries (fig 1B). Cardiac catheterisation with a pulmonary angiogram confirmed the diagnosis. Local thrombus formation, especially in the right pulmonary artery, was found (fig 2A). Severe pulmonary hypertension (94/ $49 \mathrm{~mm} \mathrm{Hg}$, mean $68 \mathrm{~mm} \mathrm{Hg}$ ) and a low cardiac output (2 l/ min) were documented. In addition, there was a filiform stenosis of the proximal left main coronary artery and total exclusion of the right upper pulmonary vein caused by local compression by the PAA (fig 2B). The stenosis on the left main coronary artery was dilated and stented because of intermittent episodes of ischaemia on serial ECGs. The patient is scheduled for heart-lung transplantation.

\section{DISCUSSION}

Aneurysms of the pulmonary artery are uncommon. In 1947 a review of 109571 necropsies was published in which only eight cases of PAAs were found. ${ }^{1}$ The first descriptions of PAAs date back to the 1700s. In the pre-antibiotic era, the majority of cases were caused by tuberculosis or syphilis. ${ }^{1}$ Nowadays, aneurysms are associated with congenital heart disease in more than $50 \%$ of cases. ${ }^{1-3}$ It appears most frequently with a patent arterial duct, an atrial septal defect, or a ventricular septal defect. Other congenital defects reported in conjunction with PAA are tetralogy of Fallot, pulmonary valve stenosis, pulmonary valve regurgitation, bicuspid pulmonary valve, and transposition of the great arteries. In the remaining reported cases, PAA is associated mainly with secondary acquired lesions such as bacterial endocarditis, trauma, septic emboli, cystic medial necrosis, mycotic aneurysms caused by other bacteria and fungi, especially Staphylococcus aureus and streptococci, ${ }^{14}$ or congenital anomalies such as Marfan's syndrome, ${ }^{4}$ congenital pulmonary arteriovenous aneurysms, ${ }^{4}$ hereditary haemorrhagic telangiectasia, or vasculitis such as Hughes-Stovin or Behçet's syndrome. ${ }^{4}$

There are several possible mechanisms of pathogenesis: weakness of the pulmonary artery wall caused by congenital structural abnormality ${ }^{4}$; increased pulmonary blood flow and pulmonary hypertension; marantic embolisation causing local vascular inflammation and breakdown; and infection with vascular seeding of the organism. ${ }^{4}$

To make the diagnosis, a high index of suspicion is first required. The clinical manifestations of PAA are diverse and non-specific. Symptoms associated with PAA are exertional dyspnoea, haemoptysis, cough, and chest pain. The presence of the PAA can be confirmed by medical imaging techniques such as conventional chest radiography, computed tomography, magnetic resonance imaging, and pulmonary angiography.

Little is known about the natural history of PAAs. However, all aneurysms have the potential to rupture and it has been suggested that a third of patients with a proximal PAA die of this type of complication. ${ }^{6}$ Haemoptysis is a marker of instability of the lesion and a strong indicator for intervention. ${ }^{46}$

Other possible complications are the compression of adjacent structures. A few cases of airway compression have been described. ${ }^{7}$ Compression of the left main coronary artery and the right upper pulmonary vein in an adult without obvious congenital cardiac disease as in our patient has never been described. Direct coronary artery compression by a PAA has only been reported in association with an atrial septal defect $^{5}$ and in isolated case reports in conjunction with a patent arterial duct, ${ }^{8}$ tetralogy of Fallot with pulmonary valve agenesis, endocardial cushion defect, ${ }^{9}$ and primary pulmonary hypertension. ${ }^{10}$ The clinical significance of compression of the left main coronary artery by a dilated pulmonary artery is not clear. According to some small studies and case reports it may be a cause of sudden death. ${ }^{58}$ 

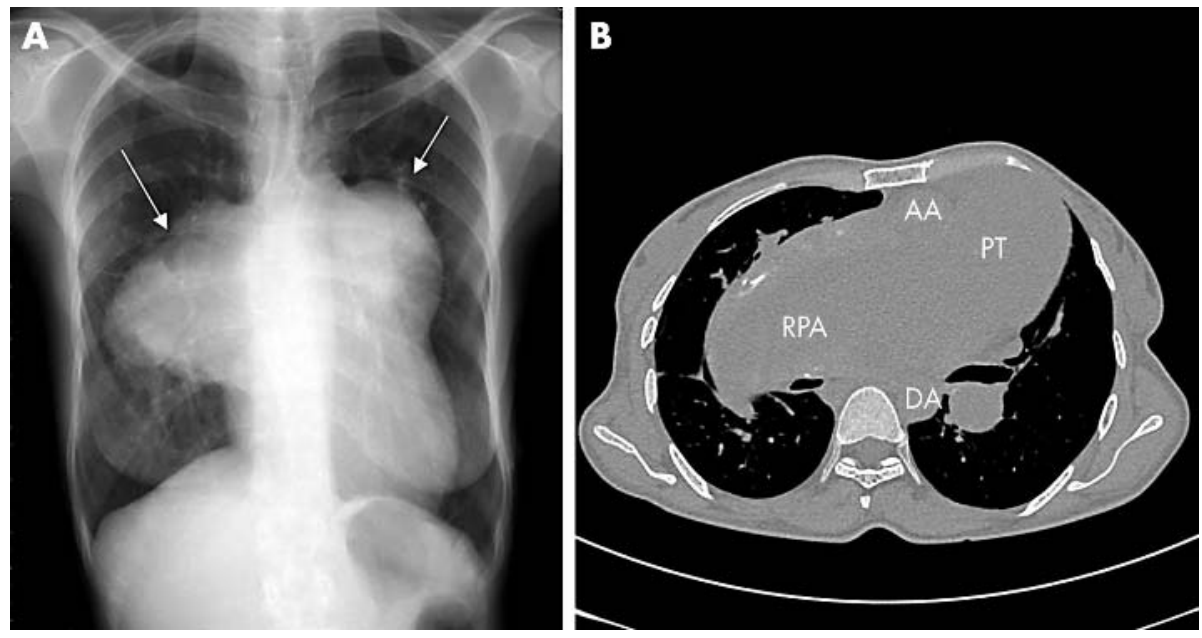

Figure 1 (A) Chest radiograph. Bilateral hilar masses were found indicating the aneurysmal dilatation of both pulmonary arteries (arrows). (B) Computed tomogram of the chest. The pulmonary trunk was characterised by aneurysmal dilatation (PT). On this slice the extension to the right pulmonary artery (RPA) of the aneurysm is documented. AA ascending aorta; $A D$ : descending aorta.
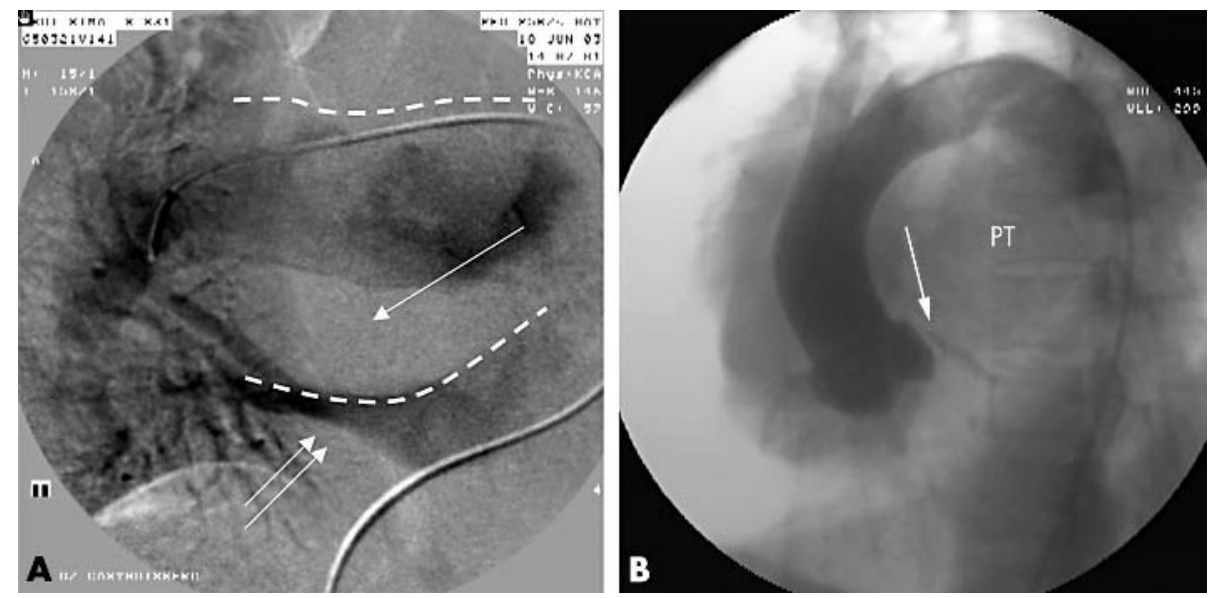

Figure 2 (A) Subtraction image of a pulmonary angiogram with levophase. The dotted lines indicate the aneurysmal deformation of the right pulmonary artery. There is no contrast filling in the dorsal part of the pulmonary artery compatible with local thrombus formation (single arrow). Only backflow of the lower right pulmonary vein was documented (double arrow). (B) Aortogram showing that the left main coronary artery had a filiform stenosis (single arrow) by compression of the pulmonary trunk (PT).

The treatment of patients with PAA is complicated and as the disease progresses the treatment alternatives become limited. There are no firm guidelines for optimal treatment of PAA. The first rule is to correct any underlying cause (for example, infection should be treated and congenital abnormalities amenable to therapeutic interventions should be corrected). The second step consists of addressing the PAA itself. Treatment options vary with the location of the aneurysm. When the main pulmonary trunk is involved, surgical intervention consists of aneurysmectomy or aneurysmorrhaphy. ${ }^{17}$ With more peripheral lesions, embolotherapy has become the treatment of choice, instead of the previously performed lobectomy. ${ }^{16}$ With advanced disease, large aneurysms develop, usually reflecting severe pulmonary hypertension, and converge in a high mortality. ${ }^{47}$ The combination of the commonly underlying cardiovascular disease makes these patients poor surgical candidates.

PAA remains a very rare condition with a high morbidity and mortality. Therefore, the clinician must have a high degree of suspicion to make the diagnosis. The advanced stage of the disease of our patient led us decide to schedule a heart-lung transplantation.

\section{Authors' affiliations}

V Decuypere, W Budts, Department of Cardiology, University Hospitals, Leuven, Belgium

M Delcroix, Department of Pneumology, University Hospitals
Correspondence to: Dr W Budts, Department of Cardiology, University Hospitals Leuven, Herestraat 49, B-3000 Leuven, Belgium; werner.budts@uz.kuleuven.ac.be

Accepted 13 November 2003

\section{REFERENCES}

1 Deterling RA Jr, Clagett OT. Aneurysm of the pulmonary artery: review of literature and report of a case. Am Heart J 1947;34:471-98.

2 Boyd U, Mc Gavack TH. Aneurysm of the pulmonary artery: a review of the literature and report of two new cases. Am Heart J 1939;18:562-78.

3 Bhandari AK, Nanda NC. Pulmonary artery aneurysm: echocardiographic features in 5 patients. Am J Cardiol 1984;53:1438-41.

4 Bartter T, Irwin RS, Nash G. Aneurysms of the pulmonary arteries [review]. Chest 1988;94:1065-75.

5 Fujiwara K, Naito Y, Higashive S, et al. Left main coronary artery compression by dilated pulmonary artery: report of three cases. $J$ Thorac Cardiovasc Surg 1992;104:449-52.

6 Pereira de Godoy JM, Batigalia F. Bilateral pulmonary artery aneurysm associated with bilateral pulmonary thromboembolism, superior vena caval thrombosis, and Chagas' disease. Angiology 2000;7:609-14.

7 Araujo CE, Rubio ER, Susanti IE, et al. Airway obstruction due to bilateral giant pulmonary artery aneurysms. South Med J 2002;95:366-8.

$8 \mathrm{Bijl}$ M, Bronzwaer JGF, Van Rossum AC, et al. Angina pectoris due to left main coronary artery compression in Eisenmenger ductus arteriosus. Am Heart $J$ 1993;125:1767-71.

9 Manojkumar R, Grover A. Left main coronary artery compression by dilated main pulmonary artery in endocardial cushion defect. Indian Heart J 2002;54:74-6.

10 Patrat JF, Jondeau G, Dabourg O, et al. Left main coronary artery compression during primary pulmonary hypertension. Chest $1997 ; 112: 842-3$. 